\title{
CONF- $950848--7$
}

\section{DSTPIBUTION OF THIS DOCUMENT IS UNMMTED JR}

\section{SPECTROSCOPY OF DIVERTOR PLASMAS}

\author{
R. C. Isler \\ Fusion Energy Division \\ Oak Ridge National Laboratory \\ Oak Ridge, TN 37831, USA
}

RECFIVO

FEB 05 พบบ

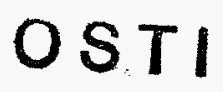

\begin{abstract}
The requirements for divertor spectroscopy are treated with respect to instrumentation and observations on present machines. Emphasis is placed on quantitative measurements.of impurity concentrations from the interpretation of spectral line intensities. The possible influence of non-Maxwellian electron distributions on spectral line excitation in the divertor is discussed. Finally the use of spectroscopy for determining plasma temperature, density, and flows is examined.
\end{abstract}

\section{INTRODUCTION}

Studies of impurities in plasma confinement devices have always constituted an integral part of fusion research efforts since it will be necessary to develop methods of controlling the core concentrations of these ions in a reactor to minimize the dilution of the D$T$ fuel and to suppress the bremsstrahlung radiation. As the fusion program moves toward power producing devices, such as ITER, it is becoming even more imperative to understand the means by which pruduction of impurities can be ameliorated and the techniques by which their transport into the core plasma may be inhibited. At present, it is envisioned that such impurity control, as well as power and particle handling, will be achieved primarily by poloidal divertors. However, the development of divertors that can deal with extreme heat loads presents a serious technological challenge because the power deposition tends to concentrate in narrow regions where the field lines strike the target plates, a situation that leads to severe demands on materials as well as to potentially large impurity production rates as a result of erosion. One solution proposed for successful operation is the creation of highdensity, highly-radiating plasmas from which power can be distrbuted through charge exchange or through spectral line emission more uniformly over the divertor region. Such situations might be realized by the deliberate injection of impurities. The current ITER designs call for $400 \mathrm{MW}$ of heating power with $300 \mathrm{MW}$ delivered to the divertor. But the power incident on the target plates must be less than $50 \mathrm{MW}$, so the strategy is to transfer up $250 \mathrm{MW}$ to the first wall and divertor chamber by atomic processes. ${ }^{1}$ In presently operating tokamaks, where the efficacy of this approach must be verified, it is anticipated that nitrogen, neon, argon, and krypton will be tested as radiating targets and that beryllium, boron, carbon, molybdenum, and tungsten will serve as plasma facing materials on target plates. 
Therefore, both from the necessity to understand intrinsic impurity production and the need to evaluate the feasibility of radiating divertors for dissipating power benignly, spectroscopy of divertor plasmas is becoming an intensive research area.

The goal of this paper is not to present specific designs for spectroscopic instrumentation in the ITER divertor but to outline the requirements that will be necessary for understanding impurity erosion and transport in light of the results that are rapidly accumulating from presently operating machines.

\section{INSTRUMENTATION}

A major goal of divertor spectroscopy in present machines is the determination of absolute concentrations and radiative losses of impurities as a function of location in order to benchmark predictive codes that can be used to model ITER operation. Unlike the main plasma where this problem can often be reduced to a one-dimensional characterization in terms of the minor radius, it is necessary to obtain rather complicated two-dimensional (or even three-dimensional) spatial distributions for divertors. An ideal experimental arrangement would incorporate multiple views over the entire vacuum ultraviolet region of the spectrum to observe the resonance lines of impurity ions (optically allowed excitations from the ground or metastable states) and to determine their distributions. The calculated excitation rates for the resonance lines are generally more accurate than for other transitions, and they are often only weakly dependent on electron temperature in the regions of interest, in contrast to lines originating from highly excited states. Morover, it is frequently impossible to detect suitable spectral lines in the visible region as a means of obyaining a comprehensive analysis of a particular impurity, i.e., to find lines which are both intense enough to be measured and for which accurate excitation rates are available. Visible spectroscopy is most useful for impurities with $\mathrm{Z} \leq 7$ (nitrogen); it is difficult even to detect neon ions other than Ne II at long wavelengths. Nevertheless, the ease of obtaining spatial resolution from visible transitions, including ones that cannot be easily interpreted in terms of densities or radiated power, still renders them valuable for understanding the functioning of divertors. Also, since the viewing access for the vacuum ultraviolet region in the ITER divertor may be quite restricted, one of the prime objectives of current research should be to establish a firm basis for analysis of visible lines if it turns out that they are the main source of divertor impurity data from future machines.

Several types of spectrometers provide various capabilities in the vacuum ultraviolet region below $2000 \AA .2$ Czerny-Turner mounts can operate from the infrared region down to almost $1150 \AA$ and are particulary useful for detecting strong transitions in Be-like and Lilike ions in carbon and lighter elements. This type of spectrometer provides nearly stigmatic images with excellent spectral resolution. Normal incidence devices can generally be employed from $6000 \AA$ to $350 \AA$, which is adequate to detect the resonance lines of all the low-Z impurities except for the helium-like and hydrogen-like species. The requirements for optics and detectors varies considerably over these wide spectral ranges, of course. Grazing incidence spectrometers can cover the region from $10 \AA$ to about $1200 \AA$, and the multichannel SPRED ${ }^{3}$ spectrometers are most useful from about $100 \AA$ to $1100 \AA$. Recent developments with multi-layer mirror ${ }^{4}$ spectrometers have also proved promising for monitoring specific spectral regions, and it is possible they could serve as compact, versatile instruments for ITER. An excellent example of employing the combination of a grazing incidence and a normal incidence spectrometer to assess divertor impurities in JT-60U can be found in Ref. 5, where comprehensive measurements of the resonance line intensities allowed the determination of the fractions of radiation attributed to deuterium, carbon, and oxygen.

The ideal array of spectrometers is difficult to implement on most machines because of the extensive spectroscopic hardware required and because views into the divertor are often quite restricted. However, it is possible to design systems that provide most of the information necessary to determine production mechanisms, erosion rates, and general features of the transport. Figure 1 illustrates, in a highly schematic fashion, the sightlines for the divertor spectrometers on ASDEX-U. A dual system consisting of a Czerny-Turner spectrometer to detect visible emissions and a normal incidence spectrometer for the vacuum ultraviolet are installed so that they view the divertor area through a movable mirror (boundary-layer spectrometer-BLS) ${ }^{6}$. This setup permits scans across the outer target plate 
and also operates to produce a limited scan in the toroidal direction. A second spectrometer array consisits of a 16 channel visible system viewing almost parallel to the face of the outer target plate and having vertical chordal resolution of $1 \mathrm{~mm}$ or alternatively, 16 channels that cover the region from $5 \mathrm{~mm}$ to $100 \mathrm{~mm}$ above the target (divertor spectrometer - DIV) 7 . These two systems combine to provide data with excellent spatial resolution in nearly orthogonal directions.

In addition to high resolution spectrometers, it is possible to employ tangentially oriented cameras equipped with narrow band filters for determining the 2-dimensional emission patterns of individual lines in the visible region of the spectrum. A diagnostic camera of this type has proven to be very useful on DIII-D 8 both for comparing carbon emissions to bolometric data and for helping to benchmark modelling codes.

\section{MODELLING AND ATOMIC PHYSICS}

Because of the complex magnetic field structure outside the last closed flux surface, quantitative analysis of spectral emissions to obtain fluxes and densities in a divertor and scrape-off layer is more difficult than the analysis of the core plasma. Neutral impurity atoms produced at the inner and outer strike points become ionized in the divertor region and are then transported along field lines. Frictional forces tend to drive them back toward the plate, but thermal-gradient forces push them toward the midplane. In general, Monte-Carlo codes, such as DIVIMP 9 or $\mathrm{MCI}^{10}$ are really needed to model the impurity behavior, nevertheless, it is useful to use a one-dimensional code like NEWT-1D 11 to gain some insight into the impurity ion distributions expected in the divertor and SOL.

Figure 2 illustrates predicted plasma parameters and radiated power deduced from the NEWT-1D code for different ionization stages of carbon as a function of distance Z measured from the midplane of the DIII-D tokamak. The target plate lies at $-136.2 \mathrm{~cm}$ and the $\mathrm{X}$-point is approximately at $-123 \mathrm{~cm}$. The simulation is based on $8 \mathrm{MW}$ of input power with sputtering at the plate as the carbon production mechanism. The details of such results depend on a variety of inputs to the code, but the general characteristics of the impurity radiation are typical of what can be expected. The electron temperature is $40 \mathrm{eV}$ at the target plate and rises to $80 \mathrm{eV}$ at the midplane. The electron density is a maximum of $9 \times 10^{13} \mathrm{~cm}^{-3}$ at $6 \mathrm{~cm}$ above the target and falls to about $1 / 2$ this value at the midplane.The radiation from the easily ionized stages, C II - C IV, is constrained to lie near the divertor floor, whereas $\mathrm{C}$ $\mathrm{V}$ and $\mathrm{C}$ VI spread upward into the scrape-off layer. The total radiation from carbon is calculated to be $285 \mathrm{~kW}$, or about $3 \%$ of the input power.

As already noted, it is preferable to have access to the vacuum ultraviolet region of the spectrum for determining the radiated power from a particular ionization stage since the resonance lines are dominant. It is not always possible to achieve this goal, however, and many interpretations must be made from visible emissions. This problem is likely to become even more severe in the future with experiments utilizing closed divertors employing relatively deep and narrow slots. The atomic physics for calculating ionization and recombination rates for low $-Z$ ions appears to be on a relatively firm footing, but some questions still exist concerning the computation of radiation rates. This is a particularly sensitive question if one relies on the weak visible lines to infer the total radiation emitted from a particular ion. Figure 3 shows a Grotrian diagram for C III (not to scale in energy). The strongest feature of carbon in the visible region is the $4650 \AA$ triplet, but only about 0.25 $\%$ of the radiated C III power comes from these lines. The radiated power from the strong lines must be modelled using a detailed-balance (collisional-radiative) calculation if only the visible signals are measured. For many transitions the data to perform such calculations is not available. Also, at present there seem to be discrepencies in different atomic data bases. The results shown in Fig. 2 were obtained using the $\operatorname{ADAS}^{12}$ data base; the same calculations using the ADPACK ${ }^{13}$ data base give results that are roughly a factor of 2 greater for the low ionization stages of carbon. The recommended cross sections or rate coefficients for various isoelectronic sequences together with estimated uncertainties are discussed in a recent overview of the available computations. ${ }^{14}$ 
In typical single null operation in DIII-D, bolometer measurements show that the total radiated power is peaked at the inner, high-field leg of the divertor near the $\mathrm{X}$-point, 15 and data from filtered tangentially viewing cameras indicate that the C III emission exhibits roughly the same structure. ${ }^{8}$ Preliminary results of modelling with the $\mathrm{MCI}$ code imply that the radiation peak near the $\mathrm{X}$-point results from the influx of neutral carbon into this area even if the source is located at the outer strike point. This radiating region apparently mitigates the power deposited on the inner target plate whereas the outer plate is observed to have a narrow hot spot where it is intersected by the field lines. With the addition of strong D2 puffing a partial detachment occurs, during which time the power delivered to the outer target plate is reduced by a factor of almost 5 , and a strongly radiating marfe-like feature forms near the X-point. Such features are commonly observed, and in ASDEX-U a completely detached high-confinement mode ( $\mathrm{CDH}$-mode) can be achieved with the simulataneous injection of neon and deuterium where pumping of the deuterium in the divertor is important for entrainment of the neon. 16

Measurements of both carbon and boron using the DIV system on ASDEX-U (Fig. 1) indicate that the Be-like and Li-like stages are narrowly peaked only a few mm above the divertor target plate 17,18 as predicted by the DIVIMP code or by NEWT-1D simulations. But scans of the BLS mirror across the face of the target plate show that the distributions of neither the boron nor the carbon line intensities can be explained completely by sputtering as a source since the emission from lines of sight passing outside of the strike point appear too great. A good fit to the carbon data has been obtained by postulating an additional flux of carbon from the pumping duct, which is presumably produced as a result of chemical erosion. Similarly, a source of boron from the walls, perhaps in the form of boron hydrides, has been suggested as a possible factor contributing to the boron observations. On the other hand, analysis of C II and C III radiation from JET shows that the number and distribution of carbon ions in the divertor can be explained almost exclusively by physical sputtering 19 ; chemical process appear to be responsible for only $10 \%$ of the $C \Pi$ ions near the target plate supposedly as a result of prompt, localised redeposition of CD4. Therefore, it appears that the details of carbon ion production may depend on characteristics specific to individual machines. A wall source is believed to be responsible for at least $50 \%$ of the carbon in the JET core plasma; 20 a similar conclusion is reached for Alcator C-Mod where it is found that the stronger divertor source is screened very efficiently. 21

The $2^{3} \mathrm{P}-2^{3} \mathrm{~S}$ transition of B IV $(2823 \AA)$ was also observed in the ASDEX-U experiments, and somewhat curiously, it was also localized close to the target plate rather than having the expected extended distribution similar to the radiation shown for the isoelectronic C V ion in Fig.2. Since the typical electron temperatures in the region of the target plate are $20-40 \mathrm{eV}$, whereas the energy required for excitation of this ion from the ground state is more than $200 \mathrm{eV}$, it has been proposed that charge exchange from neutral hydrogen is resonsible for the observations. This explanation seems unlikely, however. At the low temperatures near the target plate charge exchange of $\mathrm{B} \mathrm{V}$ and electron ionization of B IV primarily establish the relative populations. The characteristic times for charge exchange are much shorter than for electron ionization so the density of $\mathrm{B} V$ ions is low; the concentration of B IV ions is maintained by ionization from B III which has an ionization potential of only $37.9 \mathrm{eV}$. If every ionization of B IV leads rapidly to production of the 2823 $\AA$ line through charge transfer, the ratio of electron-excited to charge-exchange-excited emission is given by

$$
\mathrm{I}_{\mathrm{cx}} / \mathrm{I}_{\text {elect }}=\mathrm{S} / \mathrm{R} \text {, }
$$

where $\mathrm{S}$ is the ionization rate coefficient for $\mathrm{B} I V$ and $\mathrm{R}$ is the excitation rate coefficient computed from the collisional radiative model. If $\mathrm{S}$ is taken as the ionization rate from the ground state, this ratio is less than 0.01 for all temperatures below $50 \mathrm{eV}$. A more detailed calculation accounting for charge exchange increasing the population of the ${ }^{3} \mathrm{~S}$ metastable state, from which only $4.4 \mathrm{eV}$ is required to excite the $2383 \AA$ line, indicates no more than a $15 \%$ influence on the emission. These conclusions are substantiated by the full numeric modelling with NEWT-1D, but they.do not explain why this transition seems so peaked near the target plate. The rapid drop in temperature along the field lines should lead to a monotonically decreasing intensity despite the fact that the boron concentration may be rising 
sharply at the plate. The explaination for the observed distribution has not been firmly established, but recent theoretical work has pointed out that deviations from Maxwellian distributions can occur as electrons drift from the midplane toward the target. ${ }^{22}$ High-energy electrons, which have low collisionality, do not have time to thermalize as they do in the core plasma where they make many toroidal revolutions on the time scale for cross field drifts. At present, this seems to be a promising avenue to explore for explaining the excitation of these lines originating on highly excited levels in He-like ions.

Although most diverted tokamak experiments have concentrated on low- $\mathrm{Z}$ materials at the target plates, Alcator C-Mod uses molybdenum and some tests have been performed with a tungsten panel in ASDEX-U.23 The heavy metals have much higher sputtering thresholds than low- $Z$ materials and should be eroded very little if ion temperatures can be maintained around $10 \mathrm{eV}$. In fact, the temperatures in the divertor of Alcator C-Mod usually do not reach the molybdenum sputtering threshold in pure deuterium plasmas. Molybdenum is produced more readily when argon, having a lower sputtering threshold, is added. 20 The heavy metals present problems for spectroscopic characterization; normally the neutral species can be observed with direct viewing onto the strike points, but lines from the low ionization stages likely to be found in the divertor cannot be distinguished except perhaps for Mo II. Tungsten has been detected in the core plasma so far only by pseudocontinuua 24 around $50 \AA$ and $28 \AA$, but distinct lines from charge states between Mo XXIV and Mo XXXIV are readily observable. 25

As already noted, gas puffing of deuterium and impurities, either separately or in combination, is employed in all machines to test the radiative divertor concept. Nitrogen, neon, and argon have all been used for such experiments. This procedure can lead to a socalled detached state where the plasma near the target plates drops to temperatures of only a few $\mathrm{eV}$ and very little heat is transferred, while a marfe forms in the vicinity of the $\mathrm{X}$-point. As expected, very little radiation is observed near the target plates while ionization stages up to the lithium isoelectronic sequence are of the low- $Z$ impurites emit strongly in the marfe region. Puffing with deuterium alone and relying upon intrinsic impurities to generate the radiative losses does not produce the desired results. In JET it has been possible to achieve detachment in this fashion only in ohmic and L-mode discharges where it is possible to radiate up to $70 \%$ of the input power for carbon tragets and $85 \%$ for beryllium targets, but further puffing leads to a density limit disruption. 26 Similar attempts during H-modes showed that the ion flow to the plates was reduced between ELM's, however, the plasma reverted to L-mode when $\mathrm{P}_{\mathrm{rad}} / \mathrm{P}$ in reached approximately 50\%. In ADSDEX-U, feedback controlled puffing of both deuterium and neon permitted the achievement of stationary, completely-detached, high-confinement operation in which $90 \%$ of the power was radiated without degrading the $\mathrm{H}$-mode parameters. 16 Only small amplitude, high-frequency ELM's that transferred little power to the targets were observed. Experiments using nitrogen as the impurity showed it to have a more favorable ratio of power radiated outside the separatrix to that radiated inside than did neon, but large-amplitude compound ELM's could not be eliminated. When using argon, the fraction of radiation inside the separatrix was larger than for the other two gases employed.

\section{DIAGNOSTIC APPLICATIONS}

Aside from impurity studies per se, spectroscopy may also prove useful for determining certain properties of divertor plasmas. The most obvious application is the measurement of ion temperatures from Doppler broadening. This method is used quite successfully for the analysis of core plasma temperatures from charge-exchange excitation of hydrogen-like ions. The analysis of data from relatively cold regions of the plasma, such as near the target plates in a divertor, may not be quite so straightforward. The Zeeman splitting of the several components of a transition can be reponsible for a large fraction of broadening and, it may be necessary in some cases to account accurately for the Paschen-Back effect in order to obtain a satisfactory fit to the lineshape. Moreover, the birth distributions of velocities from sputtering or from molecular dissociation are not Maxwellian, and the low ionization stages of an impurity may not thermalize before being ionized. In such cases, the linewidth represents some measure of the average energy rather than a true temperature.

Electron temperatures are also measurable, in principle, from the ratios of $\Delta \mathrm{n}=0$ and $\Delta \mathrm{n} \neq 0$ line intensities because the excitation energies are quite different. The ratio of the $2 \mathrm{~s}$ - 
$2 \mathrm{p}$ and $2 \mathrm{~s}-3 \mathrm{p}$ transitions have been employed in JT-60U as a qualitative means of following the evolution of the electron temperature.

Stark broadening of hydrogen spectral lines in plasmas denser than those generated in tokamaks is frequently used to determine the electron density. In tokamak plasmas this technique is usually not feasible, but recent investigations in the divertor of Alcator C-Mod 28 have shown that the $n=8$ through $n=11$ transitions in the Balmer series of deuterium can be useful for obtaining electron densities from $5 \times 10^{13}-1 \times 10^{15} \mathrm{~cm}^{-3}$. Further investigations of this spectroscopic application should be pursued.

Finally, it would be highly desirable to measure impurity flows in the divertor in order to gain insight into problems of erosion and redeposition and to benchmark the codes that are being applied to predict the performance of the ITER divertor. Laser-induced fluoresence could serve as the means for such measurements. Although this diagnostic has been applied with limited success in tokamaks, continuing improvements in lasers seem to make the application promising and some preliminary experiments for developing such a diagnostic have been started on the ENCORE tokamak at the California Institute of Technology. 29

\section{Acknowledgements}

I should like to thank the following people for contributing to this presentation: $\mathrm{M}$. Fenstermacher, A. Field, J. Fuchs, R. Harvey, S. Hirshman, L. Horton A. Kallenbach, C. Klepper, K. Krieger, G. McCracken, B. Napiontek, J. Terry

\section{References}

1. D. E. Post, presented at the Workshop on Plasma Edge and Divertor Physics, Garching, Germany, May 16-17, 1995.

2. J. A. R. Samson, Techniques of Vacuum Ultraviolet Spectroscopy, John Wiley and Sons, New York, 1967.

3.R. J. Fonck, A. T. Ramsey, and R. V. Yelle, Appl. Optics 21, 2115 (1982).

4. A. P. Zwicker, S. P. Regan, M. Finkenthal, and H. W. Moos, Rev. Sci. Instrum. 61, 2786 (1990).

5. H. Kubo, T. Sugie, M. Shimada, N. Hosogane, A. Sakasai, S. Tsuji, K. Itami, N. Asakura, and K. Shimizu, Nucl. Fusion 33, 1427 (1993).

6. A. R. Field, R. Dux, G. Fussmann, C. Rempel, U. Schumacher, and U. Wenzel, Rev. Sci. Instrum. (in press)

7. B. Napiontek, private communication

8. M. E. Fenstermacher, private communication

9. P. C. Stangeby et al., Nucl. Fusion 28, 1945 (1988).

10. T. Evans, private communication.

11. R. B. Cambell, T. W. Petrie, and D. N. Hill, J. Nucl. Mat. 196-198, 426 (1992).

12. H. P. Summers, Atomic Data and Analysis Structure, User Manual, JET Joint Undertaking.

13.R. A. Hulse, Nucl. Technol./Fusion 3, 259 (1981).

14. Atomic Data and Nuclear Data Tables 57, 1-332 (1994), Edited by J. Lang.

15. A. Leonard, private communication.

16. O. Gruber et al., Phys. Rev. Lett. 74, 4217 (1995).

17 K. Krieger, H. S. Bosch., W. Eckstein, J. D. Elder, A. R. Field, G. Lieder, C. S. Pitcher, J. Roth, R. Schneider, and P. C. Stangeby, J. Nucl. Mat. 220-222, 548 (1995).

18. K. Krieger, D. Elder, A. R. Field, A. Herrmann, D. Hildebrandt, G. Lieder, B. Napiontek, C. S. Pitcher, D. Reiter, P. C. Stangeby, and W. West, Proceedings of the 22nd EPS Conference on Controlled Fusion and Plasma Physics, Bournemouth, 1995 (in press).

19. H. Y. Guo et al., Proceedings of the 22nd EPS Conference on Controlled Fusion and Plasma Physics, Bournemouth, 1995 (in press).

20. G. F. Matthews et al., J. Nucl. Mat. 196-198, 374 (1992). 
21. C. Kurz, B. Lipschultz, G. M. McCracken, M. Graf, J. Snipes, J. L. Terry, and B. Welch, MIT Plasma Fusion Center Report PFC/JA-94-013.

22. K. Kupfer, R. W. Harvey, and O. Sauter, Abstarcts of the 1995 International Sherwood Fusion Theory Conference, Incline Village, Nevada, 3-5 April, 1995.

23. W. Engelhardt, private communication

24. R. C. Isler, R. V. Neideigh, and R. D. Cowan, Physics Letters 63A, 295 (1977).

25. J. E. Rice, J. L. Terry, K. B. Fournier, M. A. Graf, M. Finkenthal, M. May, E. S. Marmar, W. H. Goldstein, and F. Bombarda, (to be published).

26. R. D. Monk et al., Proceedings of the 22nd EPS Conference on Controlled Fusion and Plasma Physics, Bournemouth, 1995 (in press).

27. C. C. Klepper, R. C. Isler, S. J. Tobin, J. T Hogan and W. R. Hess,Proceedings of the 21st EPS Conference on Controlled Fusion and Plasma Physics, Montpelier, 27 June - 1 July, pp. 1300-1303.

28. B. L. Welch, H. R. Griem, J. Terry, C. Kurz, B. LaBombard, B. Lipschultz, E. Marmar, and G. McCracken, submitted for publication.

29. J. McChesney, private communication. 


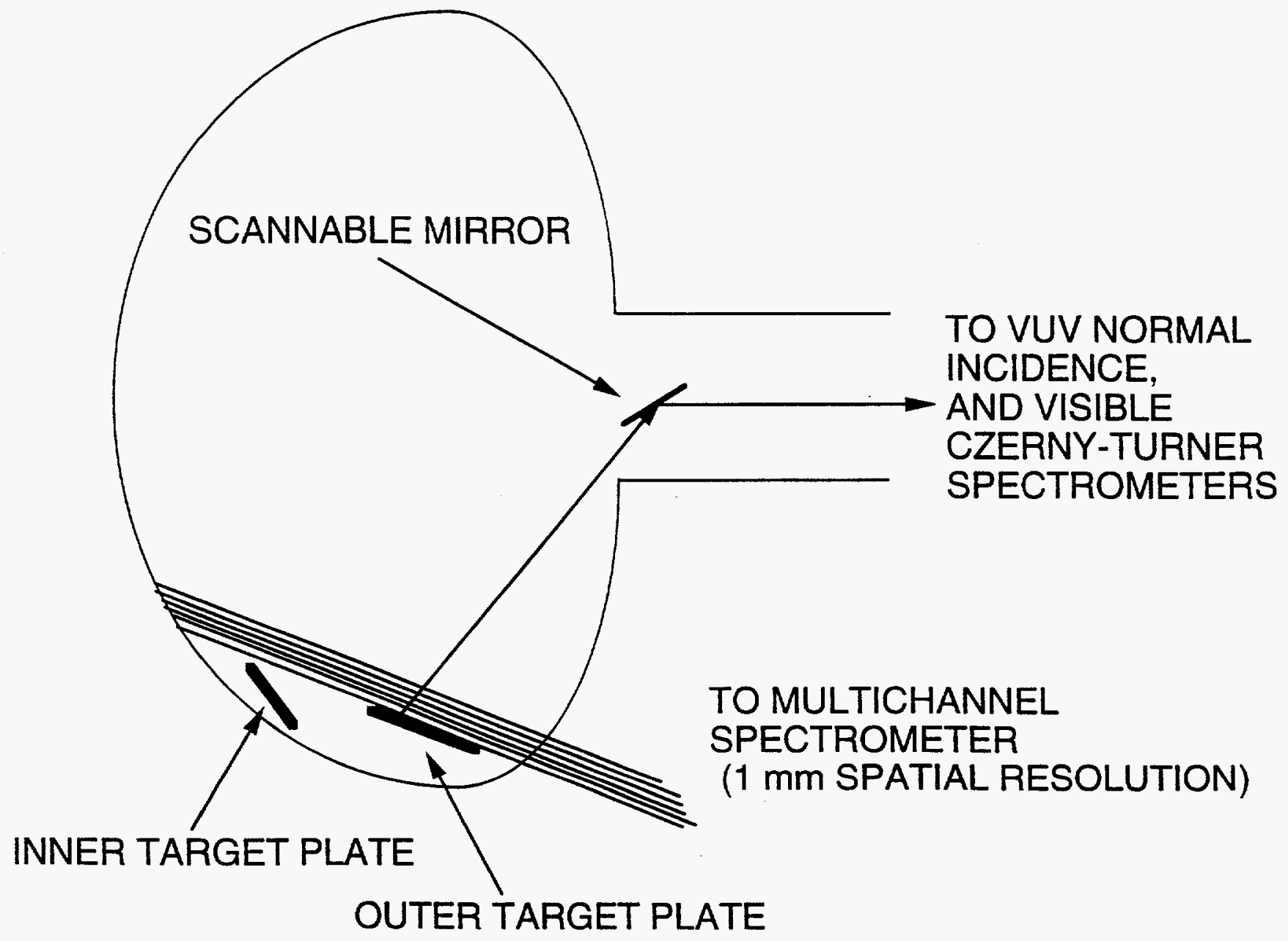

Fig. 1. Schematic of ASEDX-U showing spectroscopic views into the divertor 

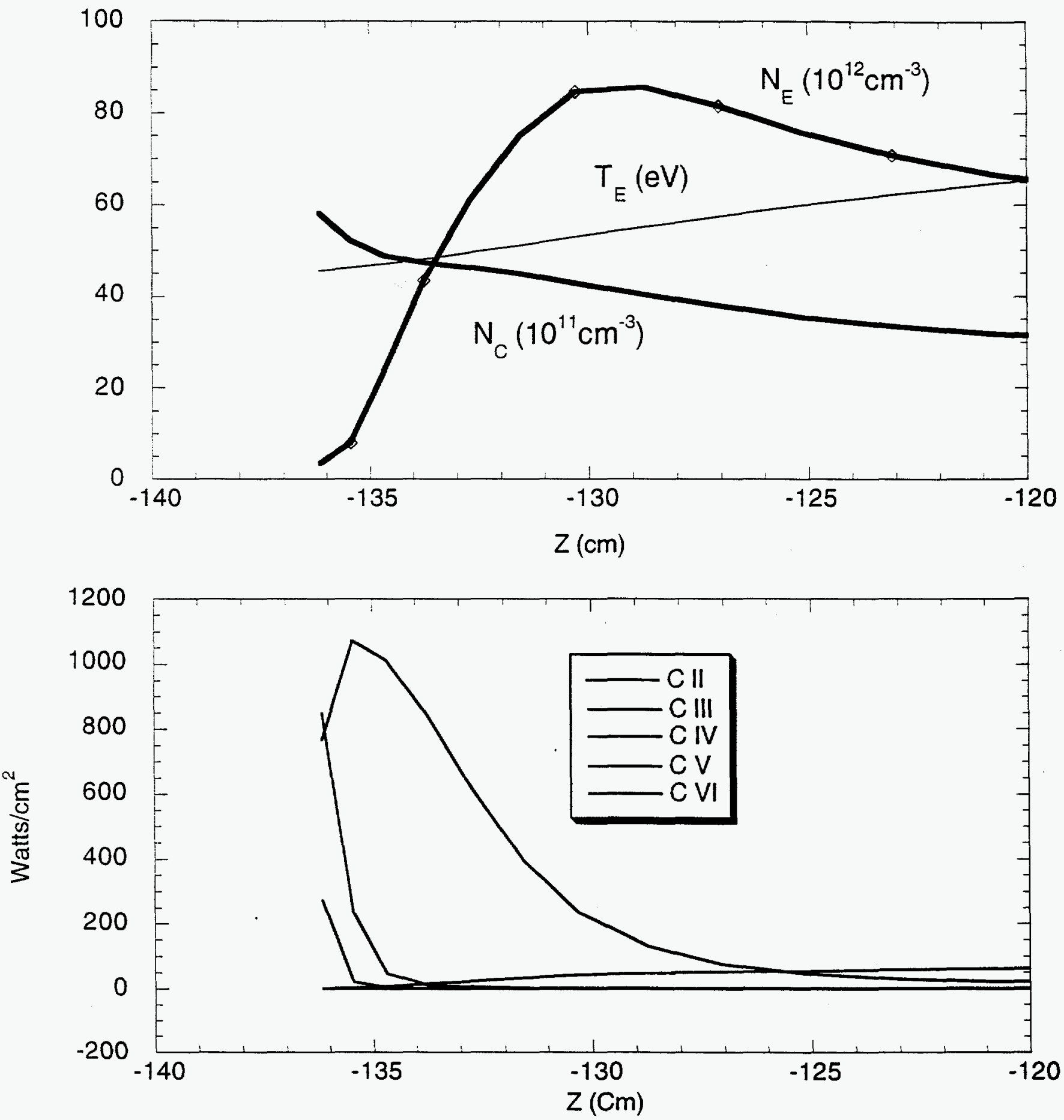

Fig. 2. NEWT-1D simulation of carbon radiation for $8 \mathrm{MW}$ of power in DIII-D. The target is at $-136.2 \mathrm{~cm}$ and the X-point at $-123 \mathrm{~cm}$. 


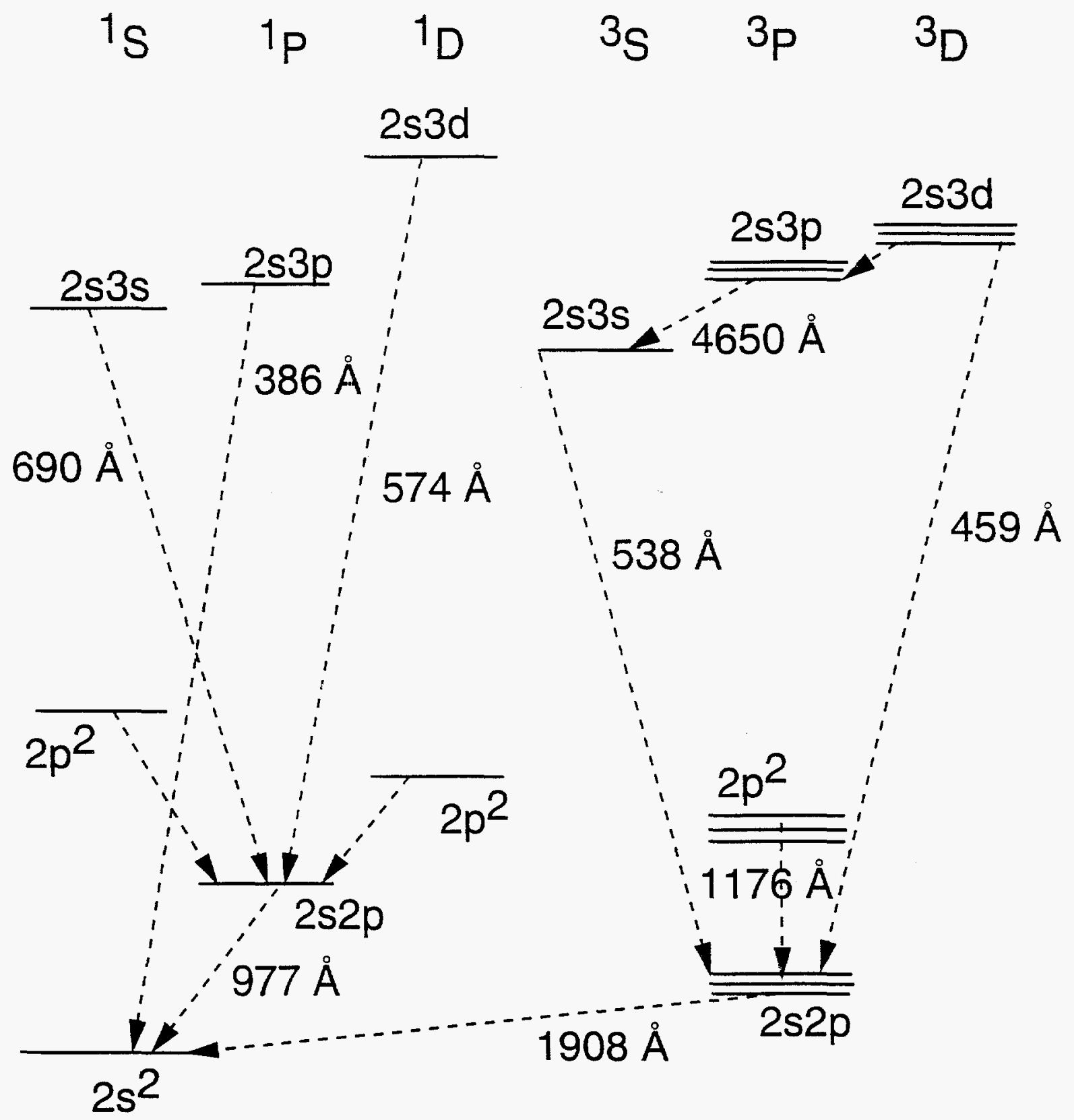

Fig. 3. Grotrian diagram for C III. 


\section{DISCLAIMER}

This report was prepared as an account of work sponsored by an agency of the United States Government. Neither the United States Government nor any agency thereof, nor any of their employees, makes any warranty, express or implied, or assumes any legal liability or responsibility for the accuracy, completeness, or usefulness of any information, apparatus, product, or process disclosed, or represents that its use would not infringe privately owned rights. Reference herein to any specific commercial product, process, or service by trade name, trademark, manufacturer, or otherwise does not necessarily constitute or imply its endorsement, recommendation, or favoring by the United States Government or any agency thereof. The views and opinions of authors expressed herein do not necessarily state or reflect those of the United States Government or any agency thereof. 
